\title{
Kearns' Illocutionary Logic and the Liar
}

In his recent paper in History and Philosophy of Logic John Kearns argues for a solution of the Liar paradox using an illocutionary logic. Paraconsistent approaches, especially dialetheism, which accepts the Liar as being both true and false, are rejected by Kearns as making no "clear sense"(51). In this critical note I want to highlight some shortcomings of Kearns' approach that fit a general dilemma for supposed solutions to the (semantic) antinomies like the Liar, a dilemma which dialetheists use to argue for their position. It is not controversial that there are languages which avoid the Liar. For example the language which consist of the single sentence "Benedict XVI was born in Germany". This language has not the resources to talk about semantics at all and thus avoids the Liar. Even more interesting languages like the propositional calculus avoid the Liar (by lacking the expressive power for semantic concepts or the means of propositional quantification). Thus a simple fact is:

(Fact) A language can avoid semantic antinomies by lacking expressive power. Kearns also agrees with the dialetheist that natural languages are semantically closed (i.e. are able to talk about their sentences and the semantic concepts and distinctions they employ):

(Claim) Natural languages are semantically closed.

Without semantic closure the Liar would be no real problem for us (speakers of natural languages). Given the (Claim) natural languages have the expressive power that may lead to the semantic antinomies. The dialetheist argues for his position by proposing a general hypothesis (cf. Bremer 2005: 27-28):

(Dilemma) A linguistic framework that solves some antinomies and is capable to express its linguistic resources is confronted with strengthened versions of the antinomies.

Thus the dialetheist claims that either some semantic concepts used in a supposed solution to a semantic antinomy are inexpressible in the framework used, thus in face of the (Claim) violating the aim of being a model of natural language, or old antinomies are sold for new ones. That some framework can avoid the antinomies by lack of expressive power is no impressive news given the (Fact). This dilemma, I claim, applies to Kearns' approach as well. 


\section{Gaps Again}

Some supposed solutions of the antinomies work by introducing truth value gaps. The Liar

( $\lambda$ ) $\lambda$ is false.

is taken to be truth valueless thus blocking the antinomic reasoning. So-called pragmatic solutions of the Liar may claim that the Liar is not well-formed or fails the felicity conditions of speech acts like assertions and thus cannot be evaluated in the first place. The idea resembles the claim that

(1) The number 7 is green.

is neither true nor false since 7 is neither in the extension of "is green" nor in the antiextension of it (say "not-green") by being the wrong kind of object for colour predication, 7 falling in a third realm of objects undetermined as to their colour. The standard reply to these ideas is that once we talk about these three partitions of objects we should be able to express that two of them are opposed to the third. Thus we should be able to say that some objects are within the partition of green objects and others are outside that partition. Thus we can say

(2) The number 7 is outside of the green objects (partition). Or - introducing a new sign "NOT" for this kind of negation, if needed - we simply say

(3) The number 7 is NOT green.

7 like all objects being NOT green by either being in the anti-extension of green (i.e. being notgreen) or being not classifiable as to colour.

The same goes for "is true". One may argue with respect to

(4) The table is true as with respect to (1). And one may counter this in the same way. A table not being an object classifiable as to its semantic value certainly is NOT true. Thus if one employs a distinction between successful statements or illocutionary acts and those like the Liar which fail the respective conditions, as Kearns does, and one reserves semantic evaluation for those expressions/acts that fulfill the conditions one should be able to say

(5) $\lambda$ is NOT true

by introducing a corresponding stronger negation "NOT", and this lead straightforwardly to a strengthened Liar like

$\left(\lambda^{\prime}\right) \quad \lambda^{\prime}$ is NOT true.

Kearns mentions gap approaches early on (54). The semantics introduced by Kearns in $\S 11$ is a paradigm case of such a three valued approach. But he does not seem to consider his approach to fall prey to strengthened Liars of the sort just discussed. This may be so, since his 
framework seems to lack the expressive resources to express the semantic concepts and distinctions it employs.

\section{Inexpressibility Again}

Kearns introduce an interesting and strong illocutionary logic. Of special importance and interest is the distinction between denial and asserting the opposite. The debate around dialetheism has also made recourse to this distinction (see the papers of Brady, Priest and Shapiro in Priest/Beall/Armour-Garb 2004), and thus for the sake of the argument it is not controversial here. In the first version of his system (\$5) Kearns has a rule that makes denials and assertions of negations equivalent. Dealing with the Liar the rule is weakened in $\S 10$, and is treated as allowing for exceptions (namely in case of antinomic statements). The crucial claim is that we can - and should - deny the Liar but need not assert the negation of the Liar then (56). Thus we can have the denial

$\dashv \mathrm{A}$

but cannot infer to

$$
\vdash \sim \mathrm{A}
$$

How can this be without limitations of expressibility? Kearns works in a framework committed to consistency and committed to sentences being true if they state "the way things are"(56). Now, if the Liar is denied, then for the sake of consistency thus understood it better be that the Liar is false/not-true or NOT true. Otherwise we would deny a true statement. Now, however, we should be able to say that the Liar is false/not-true or NOT true. Otherwise there is a fact about the world which we can infer to by consistency and $\dashv \mathrm{A}$ which we nevertheless cannot express. Thus the (Dilemma) is regained. Kearns' system in $\S 10$ is another instance of the (Fact).

\section{Why Assert the Liar?}

Apart from constructing a illocutionary framework to deal with semantic antinomies Kearns main argument seems to be that asserting a semantic antinomy like the Liar cannot but frustrate the intentions that are involved in communication and assertions and denials (§9). It is not at all clear for a dialetheist that this is so. This may be set out on another occasion - and has already been argued for by Priest, for example - but the basic ideas may be given:

1. In distinction to physical properties there is nothing incomprehensible in some object like a statement having contradictory semantic properties (like being true and false at 
the same time), these properties not getting into conflict with each other like in case of an object having the physical structure of greenness and not having it. [against (53)]

2. If there are semantic facts corresponding to truth claims it may be the point of an assertion to claim that the fact that $(\lambda)$ is true is given, and that the fact that $(\lambda)$ is false is given. [Assertions about $(\lambda)$ so can "confront" the world as Kearns demands (51).]

3. There may be two facts obtaining even though the statements which correspond to these facts are contradictory. The reason to assert each of these statements being that in a semantically closed language we have a proof for both claims about the Liar (the existence of the two proofs being relevant facts themselves to be reported).

Kearns might take recourse to his strong intuitions about (real?) negation and consistency (e.g. 51, 53, 62, 63) against dialetheism. His treatment of the Liar, nevertheless, faces the (Dilemma).

\section{References}

Bremer, M. 2005. An Introduction to Paraconsistent Logics. Bern et al.: Lang.

Kearns, J. 2007. 'An Illocutionary Logical Explanation of the Liar Paradox', History and Philosophy of Logic, 28, 31-66.

Priest, G./Beall J.C./Armour-Garb, B. 2004. The Law of Non-Contradiction. Oxford: Clarendon. 